\title{
Metal-free graphene-carbon nitride hybrids for photodegradation of organic pollutants in water
}

\author{
Bing $\mathrm{Ai}^{1,2}$, Xiaoguang Duan ${ }^{2}$, Hongqi Sun ${ }^{2 *}$ Xiang Qiu ${ }^{2}$, and Shaobin Wang ${ }^{2 *}$ \\ ${ }^{1}$ School of Chemical Engineering, Shandong University of Technology, Zibo, Shandong, \\ China P.R. \\ ${ }^{2}$ Department of Chemical Engineering, Curtin University, GPO Box U1987, Perth, WA6845, \\ Australia \\ *Corresponding authors. \\ Email: h.sun@curtin.edu.au (HS), shaobin.wang@curtin.edu.au (SW)
}

\begin{abstract}
Hybrid photocatalysts of graphitic carbon nitride $\left(\mathrm{g}-\mathrm{C}_{3} \mathrm{~N}_{4}\right)$ and reduced graphene oxide (rGO) composites were prepared in one-pot via a thermal condensation of melamine with different amounts of graphene oxide (GO). As metal-free hybrids, the prepared photocatalysts presented enhanced performances in photooxidation of both methylene blue and phenol in water solutions under various light irradiations. The level of rGO significantly affected MB photodegradation efficiencies. The introduced graphene can improve the MB adsorption and optical absorption in visible light region, therefore enables the hybrids to efficiently degrade MB under visible light with wavelengths longer than $430 \mathrm{~nm}$. The metal-free photocatalysts were also able to degrade phenol effectively and the effects of catalyst loading and initial phenol concentration were investigated. This study provided an efficient and environmentally benign photocatalyst for degradation of organic pollutants in water, with complete prevention of secondary contamination from metal-leaching.
\end{abstract}

Keywords: Photocatalysis; g- $\mathrm{C}_{3} \mathrm{~N}_{4}$; Methylene blue; Phenol; Graphene; Metal-free 


\section{Introduction}

Nowadays, worldwide attention has been drawn to develop the state-of-the-art technologies to remove toxic and hazardous chemicals in various wastewaters discharged from industries and households. The most popular Fenton process generates hydroxyl radicals $(\bullet \mathrm{OH})$ to completely decompose organic compounds into water and carbon dioxide [1, 2]. However, the traditional Fenton reaction requires large amounts of chemical reagents and suffers from the generation of excess sludge and strict $\mathrm{pH}$ requirement $(\mathrm{pH} 3)[3,4]$. The leaching problems and the associated secondary contamination still stubbornly exist in any metalbased homogeneous and heterogeneous systems in the advanced oxidative processes (AOPs) $[5,6]$.

In recent years, photocatalysis has been intensively investigated as a fascinating way for oxidative reactions without consuming other oxidants [7-10]. A variety of metal-based materials were employed as efficient photocatalysts, yet the dissolved metal ions and high cost restricted their availabilities in industrial application [11, 12]. Wang and co-workers first reported that polymeric graphitic carbon nitride $\left(\mathrm{g}-\mathrm{C}_{3} \mathrm{~N}_{4}\right)$ can act as a metal-free photocatalyst to motivate water splitting for hydrogen generation under visible light [13]. Generally, g$\mathrm{C}_{3} \mathrm{~N}_{4}$ can be synthesized by thermal condensation of precursors at $500-600{ }^{\circ} \mathrm{C}$ and it presents great thermal and chemical stabilities under ambient atmosphere and superior photoelectrochemical properties [14]. Subsequent studies indicated that g- $\mathrm{C}_{3} \mathrm{~N}_{4}$ was able to be effective photocatalysts in selective oxidation [15, 16], fuel cell [17], and solar fuel production $[18,19]$.

Various strategies were applied to modify the structure of carbon nitride by nanocasting or soft templating to form feature-ordered pores and controlled intricate morphologies [14]. Transition metals, such as Fe [20, 21], Cu [22], Ta [23], Co [24], and Ag [25, 26] were also deposited onto g- $\mathrm{C}_{3} \mathrm{~N}_{4}$ to form novel organic-inorganic composites, which strongly tuned the optical, electrical and chemical properties of $\mathrm{g}-\mathrm{C}_{3} \mathrm{~N}_{4}$, and then dramatically enhanced the photocatalysis. Yan et al. [27] reported that B-doped $g-\mathrm{C}_{3} \mathrm{~N}_{4}$ could enhance the photodegradation of rhodamine B under visible light. Wang et al. [28] successfully utilized metal-free co-dopant of boron and fluorine to modify the surface chemistry and electron states of g- $\mathrm{C}_{3} \mathrm{~N}_{4}$. The innovative photocatalyst presented superior performance for oxidation of clycloalkanes toward formation of cyclohexanone with notably high selectivity. 
The emerging graphene, a new 2-dimentional (2D) nanocarbon material with sp ${ }^{2}$ hybridized carbon lattice, has opened up a brand new field in catalysis science and technology [29, 30]. The pristine graphene possesses a large specific surface area, superb charge carriers' mobility and outstanding electrical conductivity [31]. Several studies were carried out to utilize graphene to improve the photocatalytic activity of titanium dioxide [32-34]. Both experimental results and theoretical studies revealed that the enhanced photocatalytic activity mainly derived from the chemically bonded interface between graphene and $\mathrm{TiO}_{2}$ [33, 35]. Graphene was also applied to promote metal-free sulphur photocatalyst [36]. The graphene in the composite enhances the charge transportation, facilitates the separation of photo-excited electron-hole pairs, and enables the generated electrons transferring to graphene to coordinate redox reactions [33]. Zhang et al. [37] reported that rGO can modulate the band structure of g- $\mathrm{C}_{3} \mathrm{~N}_{4}$, then significantly increase the photocurrent. Li et al. [15] reported that introduction of graphene to g- $\mathrm{C}_{3} \mathrm{~N}_{4}$ can promote the conversion and selectivity in activation of $\mathrm{O}_{2}$ for selective oxidation of secondary C-H bonds of cyclohexane. Du et al. [38] applied density functional theory to theoretically investigate the interface of graphene and $g-\mathrm{C}_{3} \mathrm{~N}_{4}$, and found significant charge transfer between them, which alters the electronic properties and extends optical absorption in the visible region. Xiang et al. [39] reported that graphene/g- $\mathrm{C}_{3} \mathrm{~N}_{4}$ hybrids demonstrated enhanced photocatalytic activity for hydrogen production under visible light. However, the graphene/g- $\mathrm{C}_{3} \mathrm{~N}_{4}$ has not been investigated in degradation of organic pollutants in water.

More recently, we reported a novel metal-free hybrid of $\mathrm{g}-\mathrm{C}_{3} \mathrm{~N}_{4} /$ carbon nanospheres for degradation of organic pollutants in water [40]. In this study, we developed an in-situ approach to synthesize g- $\mathrm{C}_{3} \mathrm{~N}_{4}$ /graphene composites via a one-pot condensation. Graphene was proven to be a promising promoter for enhancing photo-oxidation of $\mathrm{g}^{-} \mathrm{C}_{3} \mathrm{~N}_{4}$ under visible and UV-visible light for phenol and methylene blue degradation.

\section{Experimental}

\subsection{Synthesis of g- $\mathrm{C}_{3} \mathrm{~N}_{4}$ /graphene hybrids (CN-G).}

Graphene oxide (GO) was prepared by a modified Hummers method [41] and detailed procedure can be found in our previous publications $[5,6]$. To prepare the $g-\mathrm{C}_{3} \mathrm{~N}_{4} /$ graphene hybrid, $5.0 \mathrm{~g}$ of melamine were mixed with fixed amount of GO $(0.1,0.2,0.4$, and $0.6 \mathrm{~g})$ in $100 \mathrm{~mL}$ ethanol solution. The suspension was stirred and kept on a hotplate at $50{ }^{\circ} \mathrm{C}$ 
overnight to dry, then collected in a crucible and transferred in a muffle furnace. The samples were annealed with a heating rate of $10{ }^{\circ} \mathrm{C} / \mathrm{min}$ to $550{ }^{\circ} \mathrm{C}$, kept at the temperature (unless mentioned elsewise) for $1 \mathrm{~h}$, and cooled down naturally. The prepared catalysts were washed with deionized water for 3 times and denoted as CN-G-0.1, CN-G-0.2, CN-G-0.4, and CN-G0.6 (referring to the amount of GO), respectively.

\subsection{Characterization of nanocarbons}

Scanning electron microscopy (SEM, Zeiss Neon 40EsB FIBSEM) was applied to investigate the morphological information of the hybrids. Transmission electron microscopy (TEM) imaging was obtained from a JEOL-2011 TEM instrument. X-ray photoelectron spectroscopy (XPS, ESCALAB 250) was carried on with an Al-Ka source. X-ray diffraction (XRD) patterns were obtained on a D8-Advanecd X-ray diffractometer (Bruker, Germany) with Cu

K $\alpha$ radiation $\left(\lambda=1.5418 \AA\right.$ ). Micrometrics-Tristar 3000 was applied for $\mathrm{N}_{2}$ adsorption to obtain the specific surface area (SSA), pore volume, and pore size distribution. Fouriertransform infrared (FT-IR) spectra were acquired from a PerkinElmer Spectroscopy-100. The thermal gravimetric-differential scanning calorimetry (TG-DSC) was performed on a MettlerToledo-Star system in air at a rate of $10^{\circ} \mathrm{C} / \mathrm{min}$ in a range of 35 - $900{ }^{\circ} \mathrm{C}$. UV-visible diffuse reflectance spectra (UV-DRS) were acquired from a JASCO-A670 spectrophotometer. The recombination behavior of photoinduced carriers was studied by photoluminescence spectra (PL) on a Varian Cary Eclipse spectrometer at an excitation wavelength of $330 \mathrm{~nm}$.

\subsection{Photodegradation of phenol and methylene blue}

Photocatalytic efficiencies of the CN-G samples were evaluated by degradation of phenol (20 ppm) and methylene blue (MB, 10 ppm) under various irradiations provided by a MSR 575/2 metal halide lamp (575 W, Philips) with different cut-off filters. In a typical experiment, 200 $\mathrm{mL}$ phenol (or MB) solution was added with $200 \mathrm{mg}$ photocatalyst (1.0 g/L) into a doublejacket reactor with cycling water pumped from a water bath at $30^{\circ} \mathrm{C}$. During each interval, 1 $\mathrm{mL}$ of the phenol solution was withdrawn and injected into a vial though a PTFE filter $(0.45$ $\mu \mathrm{m}, 25 \mathrm{~mm})$. The sample was analyzed on a Varian high performance liquid chromatography (HPLC) with a UV detector at $270 \mathrm{~nm}$ through a C-18 column. The MB suspension solution was centrifuged to remove the solid catalysts and tested on a JASCO UV-vis spectrophotometer (664 nm).

\section{Results and discussion}




\subsection{Physicochemical properties of the materials}

The morphology of the hybrids was revealed by SEM and TEM imaging. Pristine g- $\mathrm{C}_{3} \mathrm{~N}_{4}$ in Fig. 1(a) showed a slate-like bulk structure with sharp edges. Fig. 1(b) presents the typical wrinkled lamellar-like layers of reduced graphene oxide (rGO). It can be seen in Fig. 1 (c) that, after hybridization with the GO by an in-situ method, g- $_{3} \mathrm{~N}_{4}$ formed a smaller, packed and porous structure. In Fig. 1(d), rGO sheets were observed and demonstrated a close interaction with g- $\mathrm{C}_{3} \mathrm{~N}_{4}$ particles, indicating that the interfacial surfaces were formed between the rGO and $g-\mathrm{C}_{3} \mathrm{~N}_{4}$.

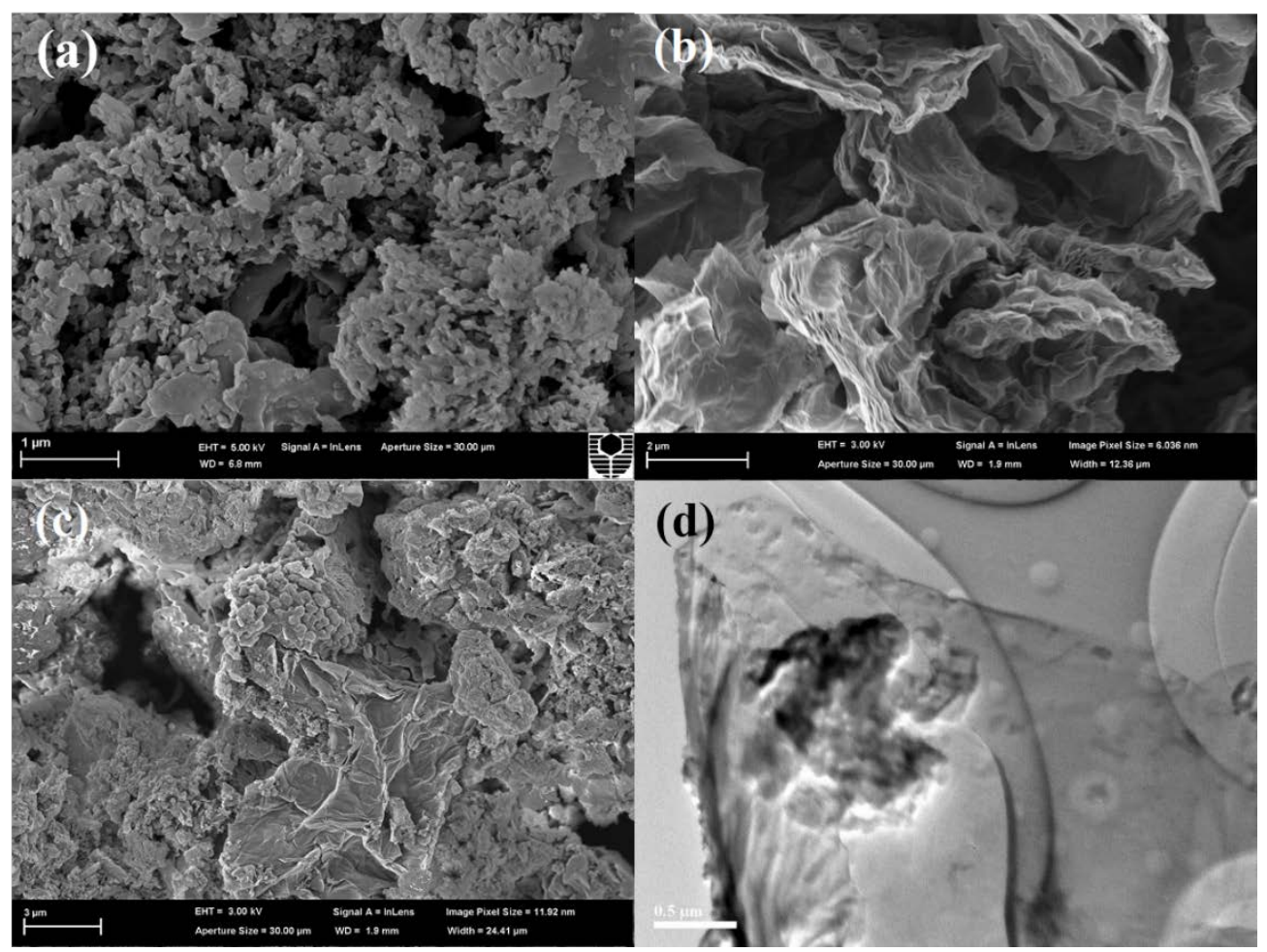

Fig. 1 SEM images of (a) g- $\mathrm{C}_{3} \mathrm{~N}_{4}$, (b) rGO-550, (c) CN-G-0.4, and (d) TEM image of CN-G0.4 .

The crystal structures of pristine g- $\mathrm{C}_{3} \mathrm{~N}_{4}(\mathrm{CN})$ and $\mathrm{CN} /$ graphene composites were studied by XRD (Fig. 2). The sharp peak at $2 \theta$ of $27.5^{\circ}$ reflects an interlayer distance of $0.324 \mathrm{~nm}$, which is referred to the stacking of $\mathrm{C}-\mathrm{N}$ bonds in the aromatic structure and reflects the (002) plane of graphitic materials [39]. The peak at $2 \theta$ of $13.1^{\circ}$ can be indexed to be (001) plane $(0.676 \mathrm{~nm})$, related to the small tilt angularity and in-plane repeated units and reported in many references in polymerized preparation of $\mathrm{CN}$ with different precursors such as urea, cyanamide, and dycyanamide [8, 40, 42, 43]. No obvious peaks for GO or rGO were found in the $\mathrm{CN}-\mathrm{G}$ hybrids. This can be ascribed to the fact that the graphene stacking was destroyed 
during the thermal annealing and reduction process, and that the peak of rGO at around $26^{\circ}$ was overlapped by g- $\mathrm{C}_{3} \mathrm{~N}_{4}$ [39].

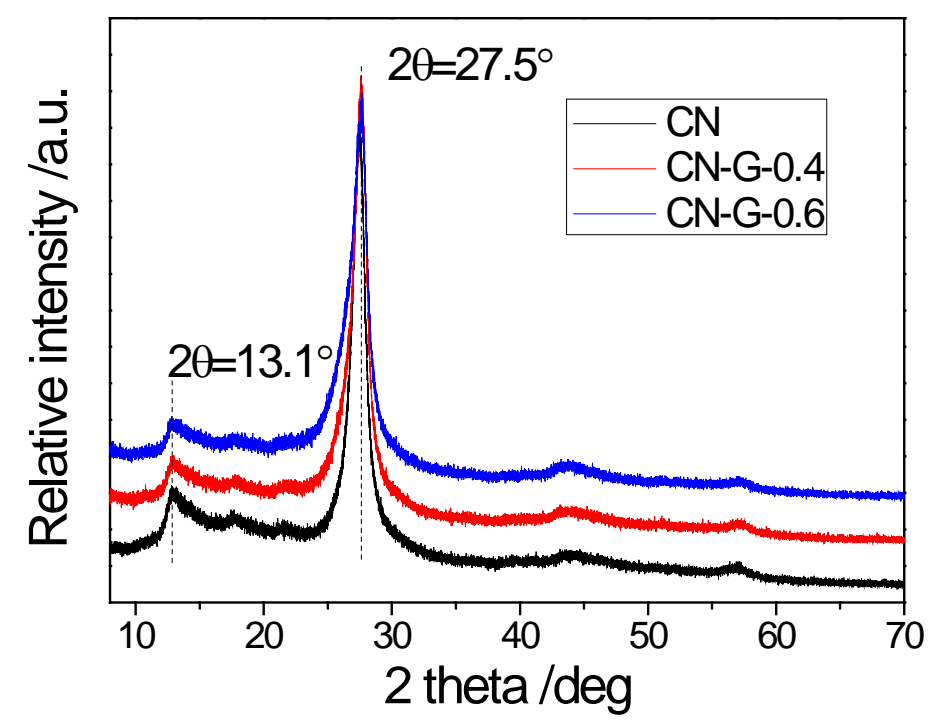

Fig. 2 XRD patterns of $\mathrm{CN}$ and $\mathrm{CN}-\mathrm{Gs}$.

The thermal stabilities of photocatalysts were evaluated by thermal analysis. It can be seen from TGA curves in Fig. 3(a) that g- $\mathrm{C}_{3} \mathrm{~N}_{4}$ demonstrated a great stability up to $550{ }^{\circ} \mathrm{C}$. It was well reported that the g- $\mathrm{C}_{3} \mathrm{~N}_{4}$ prepared at high-temperature condensation presented a stable tri-s-triazine structure [44-46]. The rapid weight loss was attributed to breaking-up of C-N bond and combustion in air. Compared to pristine $\mathrm{g}-\mathrm{C}_{3} \mathrm{~N}_{4}$, the stability of $\mathrm{CN}-\mathrm{G}$ composites decreased due to the breaking down of rGO carbon skeleton. The lower thermal stability of the chemically derived graphene was ascribed to the various defective edges and functional groups, which could act as combustion sites in air at higher temperatures. The collapse progress of the graphene network might influence the stability of CN-G hybrids. Fig 3(b) illustrates DSC curves of CN and CN-G samples. The highly-polymerized CN presented a broad endothermic peak owing to the high bonding energy of C-N bond. The exothermic peak of $\mathrm{CN}$ at $712{ }^{\circ} \mathrm{C}$ shifted to lower temperature for the hybrids and the intensity increased due to the decomposition of rGO. 

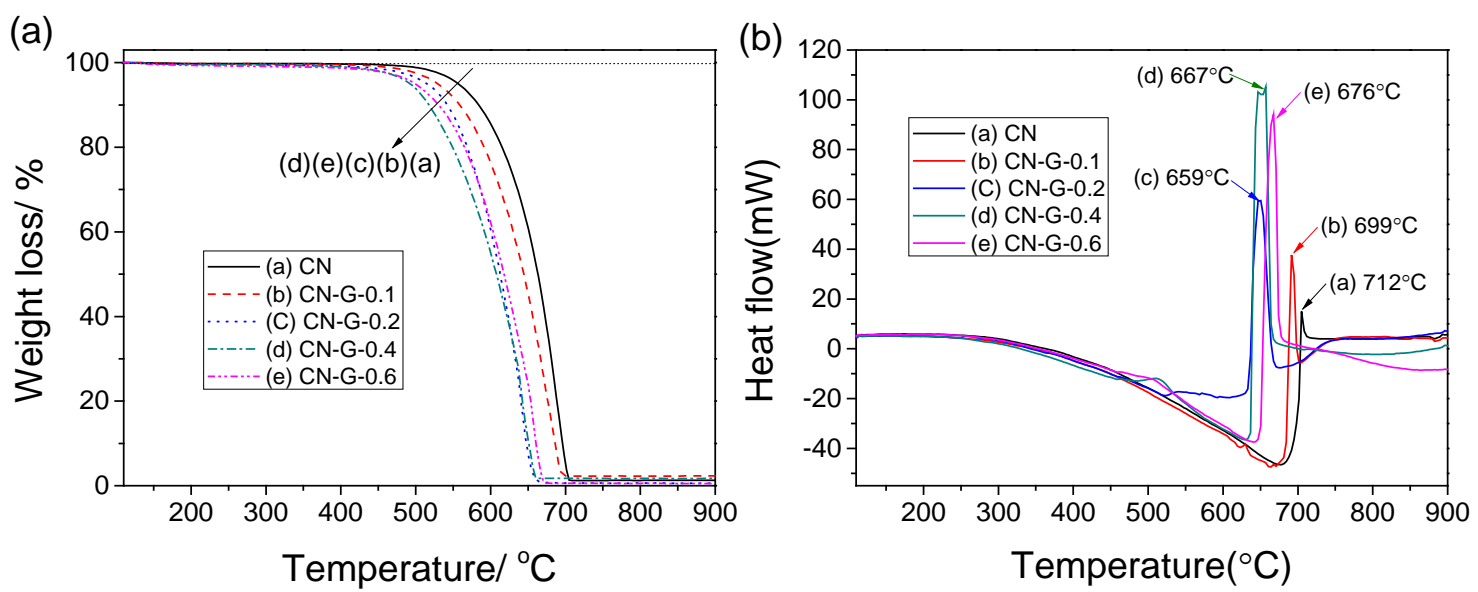

Fig. 3 TG-DSC profiles of CN and CN-G samples, (a) TGA, (b) DSC.

A comparison of FT-IR spectra between pure g- $\mathrm{C}_{3} \mathrm{~N}_{4}$ and its hybrid photocatalysts were illustrated in Fig. 4. No obvious differences were observed for the CN and CN-G-0.4. The broad peak between $3000-3500 \mathrm{~cm}^{-1}$ is associated with the adsorption of $\mathrm{H}_{2} \mathrm{O}$ and $\mathrm{CO}_{2}$ in the air [39]. Several strong bands in the region of $1200-1700 \mathrm{~cm}^{-1}$ were found in both samples. These characteristic bands arise from the typical stretching modes of $\mathrm{C}-\mathrm{N}$ heterocycles and are universally found in the g- $\mathrm{C}_{3} \mathrm{~N}_{4}$ and g- $\mathrm{C}_{3} \mathrm{~N}_{4} @$ @nanocarbon hybrids [39, 40, 47-49]. However, no $\mathrm{C}-\mathrm{O}$ or $\mathrm{C}=\mathrm{O}$ bonds were identified in the $\mathrm{CN}-\mathrm{G}-0.4$, suggesting that most of the oxygen functional groups on GO were removed during the thermal reduction. The obvious peak at around $800 \mathrm{~cm}^{-1}$ is assigned to the typical triazine-units breathing mode [39, 50].

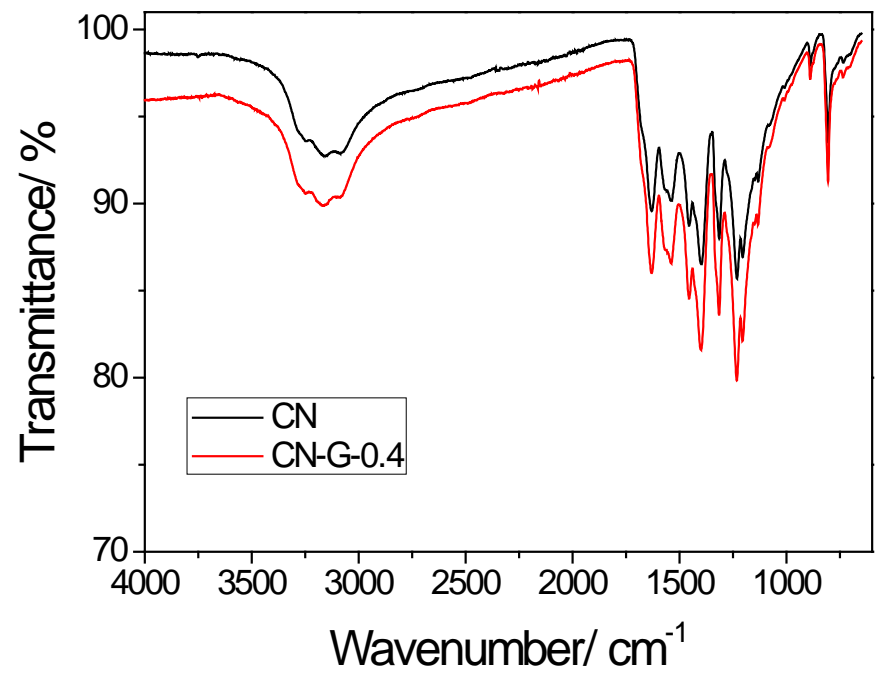

Fig.4 FT-IR spectra of CN and CN-G-0.4. 
Fig. 5(a) shows XPS survey spectra of CN and CN-G-0.4. Carbon, nitrogen and oxygen contents in pure g- $\mathrm{C}_{3} \mathrm{~N}_{4}$ is $48.03,50.89$ and 1.08 at.\%, respectively. As the carbon nitride was thermally polymerized in the muffle furnace with static air, it was likely to form some oxygen groups on the edges of the g- $\mathrm{C}_{3} \mathrm{~N}_{4}$. The chemical composition of CN-G-0.4 was much similar to $\mathrm{CN}$, suggesting that only little amount of rGO remained in the hybrids after thermal annealing. The oxygen contents increased to 1.64 at.\% on CN-G-0.4 owing to the oxygen functional groups in rGO. It was suggested that phenol and quinone groups decomposed between 500 and $900{ }^{\circ} \mathrm{C}$ and the oxygen groups were completely removed at $\sim 1100{ }^{\circ} \mathrm{C}$ [51].
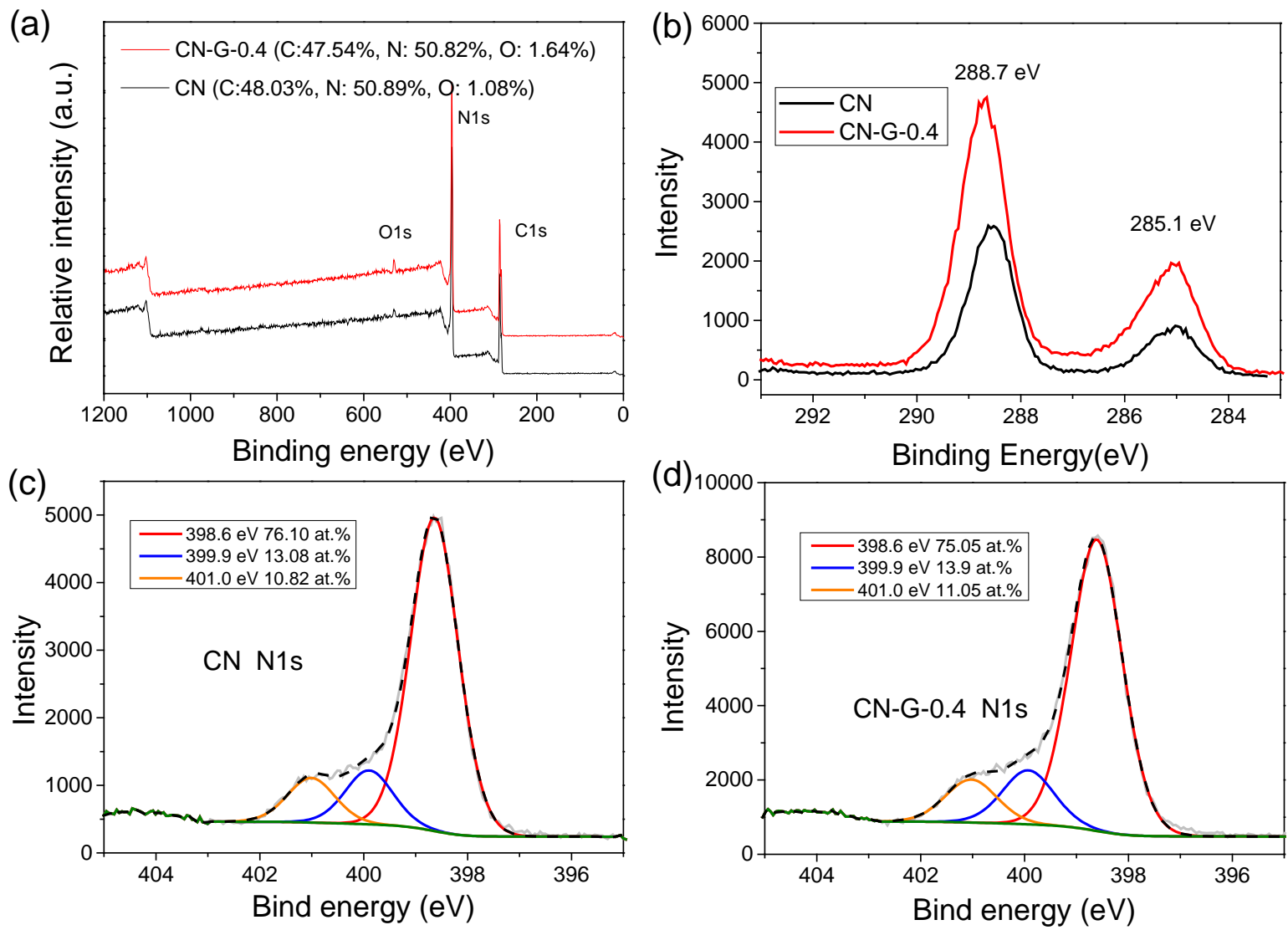

Fig. 5 (a) XPS survey of CN and CN-G-0.4, (b) C1s scan of CN and CN-G-0, (c) N1s high resolution scan of $\mathrm{CN}$ and (d) N1s high resolution of CN-G-0.4

Fig. 5(b) shows that the C1s peaks consist of two parts. The peak at the bonding energy of $285.1 \mathrm{eV}$ is corresponding to the $\mathrm{sp}^{2} \mathrm{C}-\mathrm{C}$ bond and the other sharp peak at $288.7 \mathrm{eV}$ is attributed to the carbon adjacent with three $\mathrm{N}$ atoms [39]. High resolution N1s XPS was performed to probe the $\mathrm{N}$ categories on the catalysts. The N1s peak (Figs. 5(c) and (d)) can be fitted into three parts located at 401.0, 399.9, and $398.6 \mathrm{eV}$. The peak at $398.6 \mathrm{eV}$ is associated with the $\mathrm{sp}^{2}$ hybridized $\mathrm{N}$ bonded with two carbon atoms $(\mathrm{C}-\mathrm{N}=\mathrm{C})$, which is the dominant nitrogen species in the g- $\mathrm{C}_{3} \mathrm{~N}_{4}$ with tri-s-triazine structure [52]. The other two 
peaks, 401.0 and $399.9 \mathrm{eV}$, could be assigned to the quaternary $\mathrm{N}$ ( $\mathrm{N}$ bonded with three carbon atoms, $\left.\mathrm{N}-(\mathrm{C})_{3}\right)$ and amino groups $(\mathrm{C}-\mathrm{N}-\mathrm{H})$, respectively [39, 52, 53].

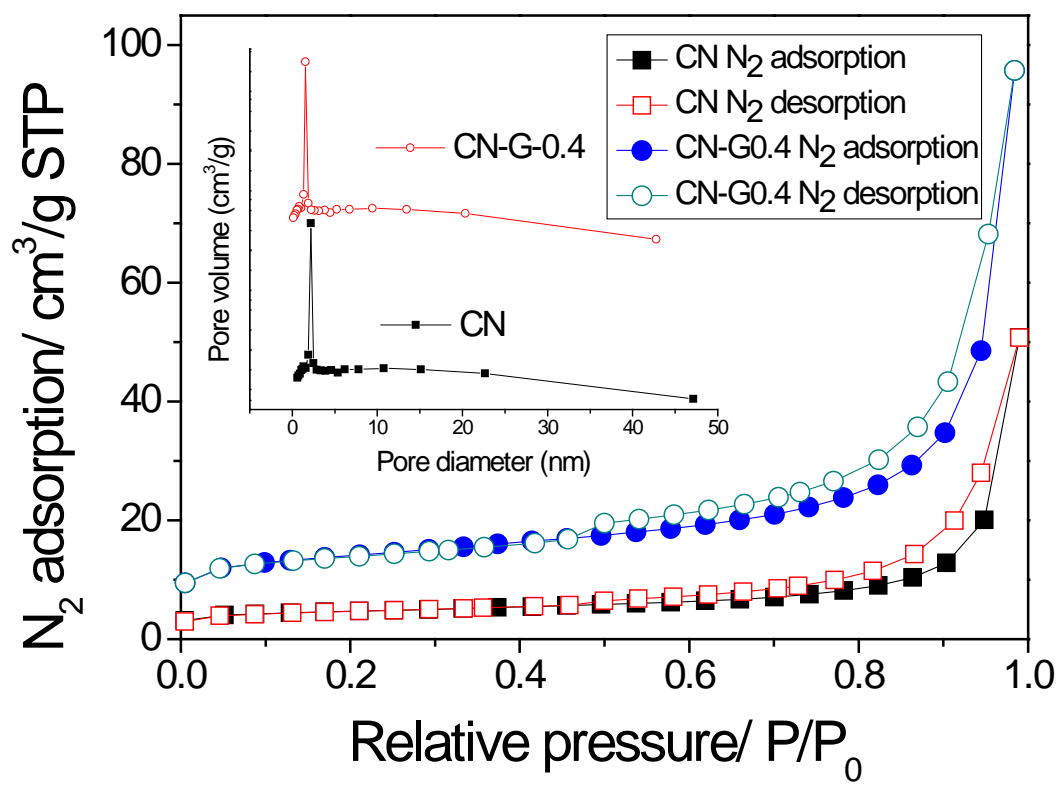

Fig. $6 \mathrm{~N}_{2}$ sorption and pore size distribution of CN and CN-G-0.4.

The pore structures and specific surface area (SSA) of CN and CN-Gs were investigated by $\mathrm{N}_{2}$ adsorption and desorption isotherms. Fig. 6 indicates mesoporous structure of the carbon catalysts $(2-50 \mathrm{~nm})$. The CN/graphene exhibits higher pore volume $\left(0.118 \mathrm{vs} 0.067 \mathrm{~cm}^{3} / \mathrm{g}\right.$ of $\mathrm{CN}$ ) and larger SSA (44.63 vs $14.73 \mathrm{~m}^{2} / \mathrm{g}$ of $\mathrm{CN}$ ). But the porous hybrids present a smaller pore size.

Fig. 7 shows $\mathrm{UV}$-vis DRS of $\mathrm{CN}$ and $\mathrm{CN}-\mathrm{G}$ samples. The pristine g- $\mathrm{C}_{3} \mathrm{~N}_{4}$ presents an absorption edge at $465 \mathrm{~nm}$ associated with a band gap energy of $2.67 \mathrm{eV}$, which is in good agreement with reported value [40]. As shown in the inset, pristine g- $\mathrm{C}_{3} \mathrm{~N}_{4}$ was bright yellow, and the introduction of rGO to $\mathrm{CN}$ would make the color changed to brown, dark blue and black at increasing rGO loadings. Accordingly, the red-shift derived from hybridization of rGO was observed. The absorption thresholds of CN-G-0.1, CN-G-0.2, CN-G-0.4 and CN-G0.6 were 494.0, 494.2, 509.8 and $533.0 \mathrm{~nm}$, respectively, and the band gap energies were then evaluated to be 2.51, 2.51, 2.43 and $2.33 \mathrm{eV}$, respectively. The results suggested that graphene can effectively increase the absorption in visible light region. 


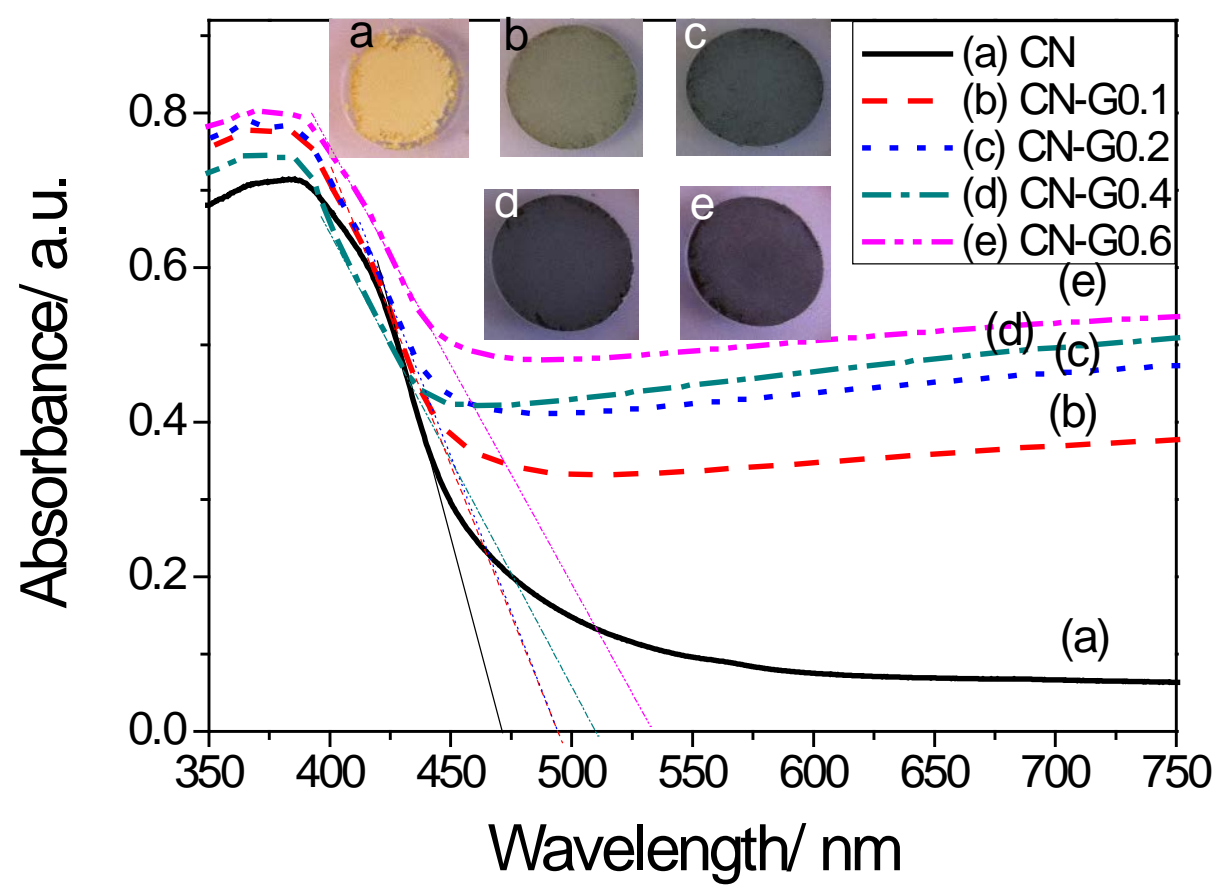

Fig. 7 UV-vis DRS and photos (inset) of the CN and CN-G photocatalysts.

Apart from the light absorption, the photodegradation efficiency is also controlled by the separation rate of the photoinduced carriers $[40,54]$. Fig. 8 shows the PL spectra of pristine and modified $g-\mathrm{C}_{3} \mathrm{~N}_{4}$ samples. The pristine $\mathrm{g}-\mathrm{C}_{3} \mathrm{~N}_{4}$ showed the highest intensity of photoluminescence, indicating the strongest recombination of carriers and the lowest photocatalytic activity. CN-G-0.1, 0.2 and 0.4 samples showed similar carries recombination, and CN-G-0.6 presented the lowest intensity.

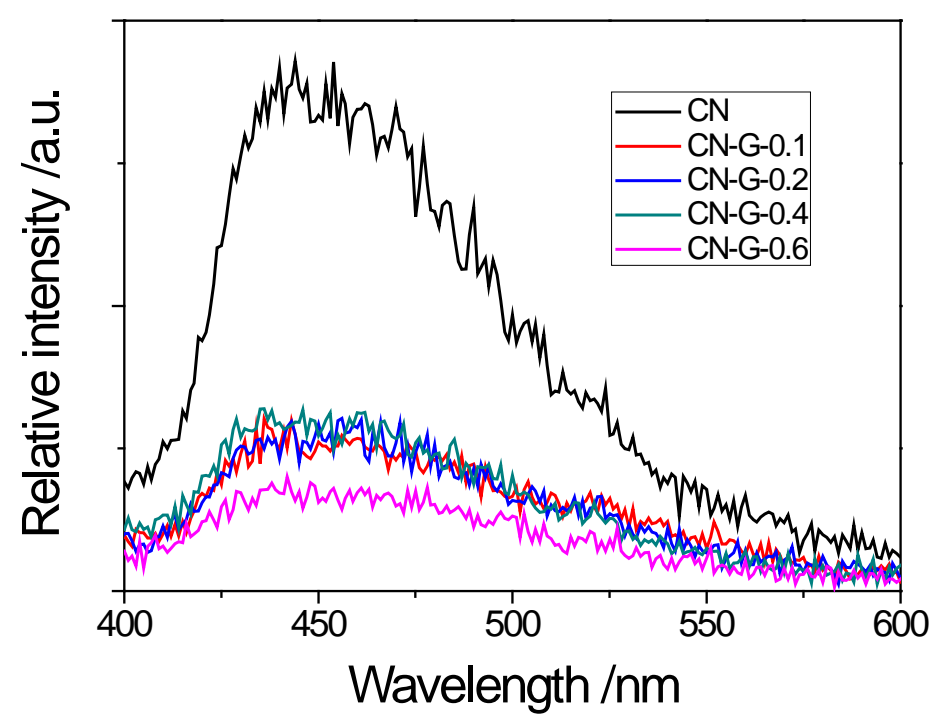


Fig. 8 PL spectra of the CN and CN-G photocatalysts.

\subsection{Photocatalytic oxidation of MB and phenol}

Fig. 9 (a) shows control experiments of $\mathrm{MB}$ and phenol adsorption on G-CN-0.4 and photolysis of MB without a catalyst. MB could hardly be degraded under UV-visible light without a catalyst. The adsorption tests indicated that about $27 \%$ of $\mathrm{MB}$ and $4 \%$ of phenol were removed due to adsorption on CN-G-0.4. MB is a cationic dye and tends to be adsorbed on the porous materials, especially on the nanocarbons with oxygen functional groups.
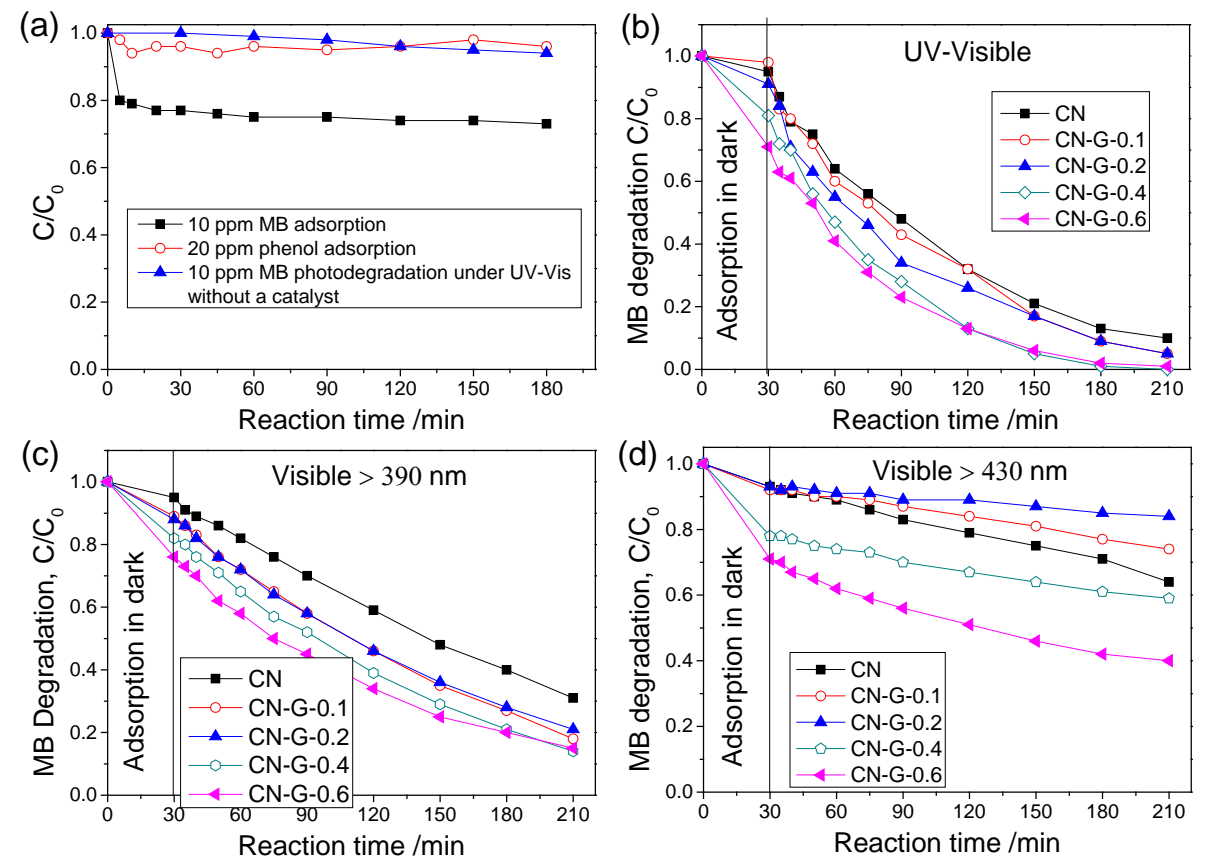

Fig. 9 Control experiments of adsorption on CN-G-0.4 and photolysis of MB under UVvisible light without a catalyst (a), and photodegradation of MB under various conditions: UV-visible light (b), visible light $>390 \mathrm{~nm}$ (c), and (d) visible light $>430 \mathrm{~nm}$. [Catalyst $=0.5$ $\mathrm{g} / \mathrm{L} ; \mathrm{MB}=10 \mathrm{ppm}$; Temperature $\left.=30{ }^{\circ} \mathrm{C}\right]$.

Fig. 9 (b) shows the effect of rGO contents on adsorption and photodegradation of MB under UV-vis irradiations. Pristine g- $\mathrm{C}_{3} \mathrm{~N}_{4}$ showed minor adsorption of $\mathrm{MB}$ and only $3 \% \mathrm{MB}$ was adsorbed in 30 min. Introduction of rGO significantly improved the MB adsorption of CN-G samples, and higher content of rGO led to higher adsorption. About $20 \%$ and $30 \%$ of MB were removed in 30 min by adsorption on CN-G-0.4 and CN-G-0.6, respectively. Around 90\% of $\mathrm{MB}$ was decomposed on pristine $\mathrm{g}-\mathrm{C}_{3} \mathrm{~N}_{4}$ by adsorption and $180 \mathrm{~min}$ photodegradation. The MB removal increased to $95 \%$ on CN-G-0.1 and CN-G-0.2 and 100\% MB removal was achieved on CN-G-0.4 and CN-G-0.6. Fig. 9 (c) shows visible light photodegradation of MB 
under light of $\lambda>390 \mathrm{~nm}$. Roughly, $69 \%$ of $\mathrm{MB}$ was removed by $\mathrm{g}^{-} \mathrm{C}_{3} \mathrm{~N}_{4}$ and $82 \%, 79 \%$, 86\%, and 85\% MB removals were achieved on CN-G-0.1, $-0.2,-0.4$, and -0.6 accordingly. Compared with the pristine g- $\mathrm{C}_{3} \mathrm{~N}_{4}$, enhanced $\mathrm{MB}$ photodegradation under visible light was observed on CN/graphene hybrids. Fig. 9 (d) shows the activities of the CN-G samples under visible light with wavelength longer than $430 \mathrm{~nm}$. Approximate 36\% MB decomposition was achieved on g- $\mathrm{C}_{3} \mathrm{~N}_{4}$ in $210 \mathrm{~min}$, whereas only $26 \%$ and $16 \%$ of MB were removed by CN-G0.1 and CN-G-0.2, respectively. Meanwhile, CN-G-0.4 and CN-G-0.6 provided $41 \%$ and $60 \%$ MB degradation, respectively. It was reported that increasing graphene contents could enhance both $\mathrm{MB}$ adsorption and visible light absorption, and then possibly improve the photocatalytic activity [38]. It can been seen in Fig 9(d) that the improved MB removal efficiency under visible light (> $430 \mathrm{~nm}$ ) was mainly due to the enhanced MB adsorption with high graphene contents owing to the strong $\pi-\pi$ interaction between MB and graphene network. When the graphene content is low, MB adsorption and optical absorption cannot be significantly improved. The complicated processes involving adsorption, light absorbance, microstructure and surface feature led to a lower MB removal on CN-G-0.1 and CN-G-0.2 than pristine $\mathrm{CN}$.

(a)

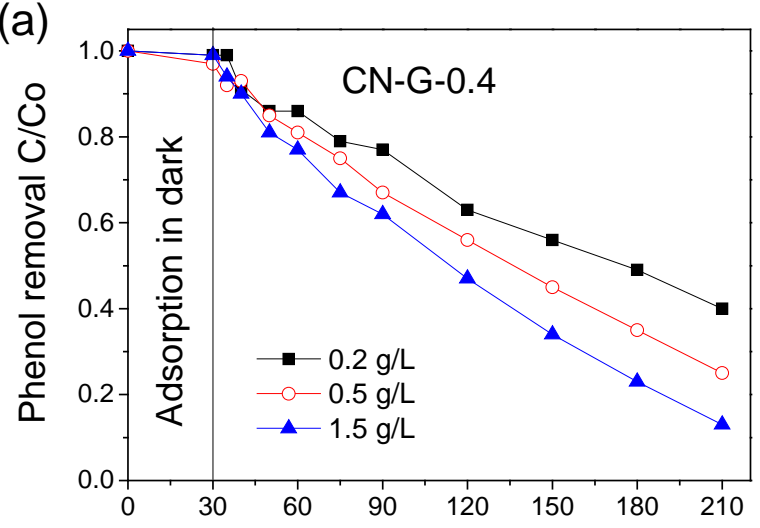

(c)

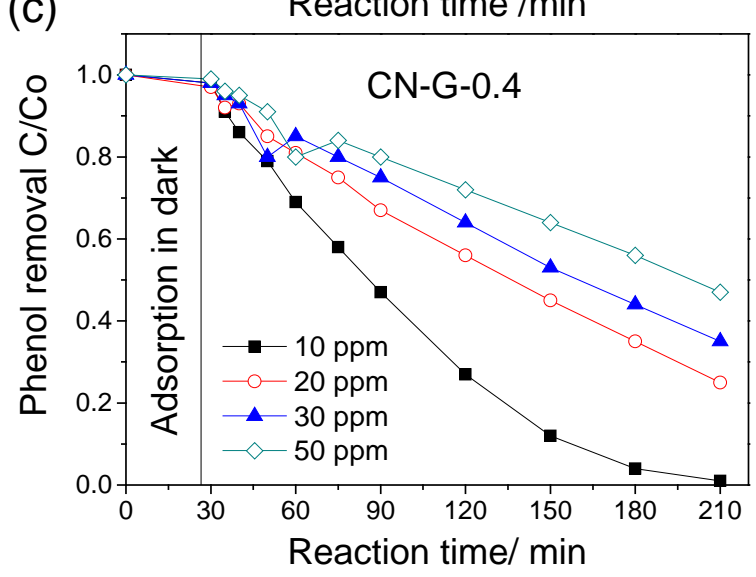

(b)
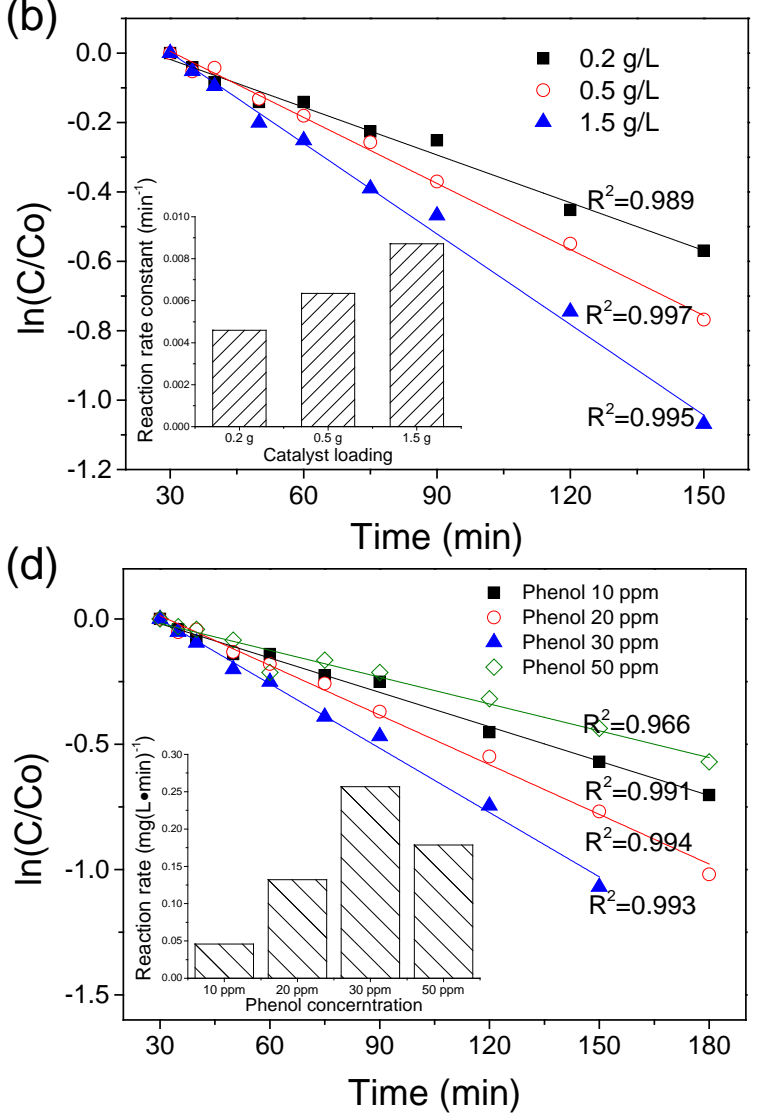

Fig. 10 Effect of catalyst loading on phenol photodegradation ((a) and (b)), [Phenol = 20 ppm; 
Temperature $=30{ }^{\circ} \mathrm{C}$; Irradiation: UV-visible light], and effect of initial phenol concentration on phenol photodegradation (c and d). [Catalyst $=0.5 \mathrm{~g} / \mathrm{L}$; Temperature $=30{ }^{\circ} \mathrm{C}$; Irradiation: UV-visible light].

CN-G-0.4 was further applied to photodegrade phenol under UV-visible light. Fig. 10(a) indicates that phenol could barely be removed by adsorption on CN-G-0.4. However, catalyst loading poses significant influence on phenol removal. Around $60 \%$ phenol removal was achieved in $210 \mathrm{~min}$ at a catalyst dosage of $0.2 \mathrm{~g} / \mathrm{L}$. While, $75 \%$ and $87 \%$ of phenol were degraded when the catalyst loading was increased to 0.5 and $1.5 \mathrm{~g} / \mathrm{L}$, respectively. The apparent reaction rate constants (Fig. 10(b)) under different catalyst loading of 0.2, 0.5, and $1.5 \mathrm{~g} / \mathrm{L}$ were estimated to be $4.59 \times 10^{-3}, 6.35 \times 10^{-3}$, and $8.71 \times 10^{-3} \mathrm{~min}^{-1}$, respectively. Increased catalyst loading produced more photo-generated active sites, which dramatically enhanced the phenol degradation efficiency. The effect of initial phenol concentration was investigated and shown in Fig. 10(c). The phenol removal efficiency decreased with increasing initial phenol concentration. Phenol could be completely decomposed in $210 \mathrm{~min}$ under a low initial concentration (10 ppm), whereas 75\%, 65\% and 53\% phenol were removed when initial concentration was increased to 20,30 , and $50 \mathrm{ppm}$. The reaction rates with initial phenol concentrations of $10,20,30$ and 50 ppm were calculated to be 0.046, 0.132, 0.257 and $0.178 \mathrm{mg}(\mathrm{L} \times \mathrm{min})^{-1}$, respectively. Fig. $10(\mathrm{~d})$ shows that the reaction rates climbed up with increased initial phenol levels from 10 to $30 \mathrm{ppm}$ and dropped when the initial phenol concentration reached $50 \mathrm{ppm}$. Increasing initial phenol levels in a proper range was able to increase the reaction rate as the phenol was quickly decomposed by the photoproduced holes and prevented the recombination. However, the excess phenol in aqueous solution would be adsorbed on the surface of the catalysts and weaken the photo-activation processes. The finite active sites generated from the fixed amount of photocatalyst could be another limiting factor as well. 


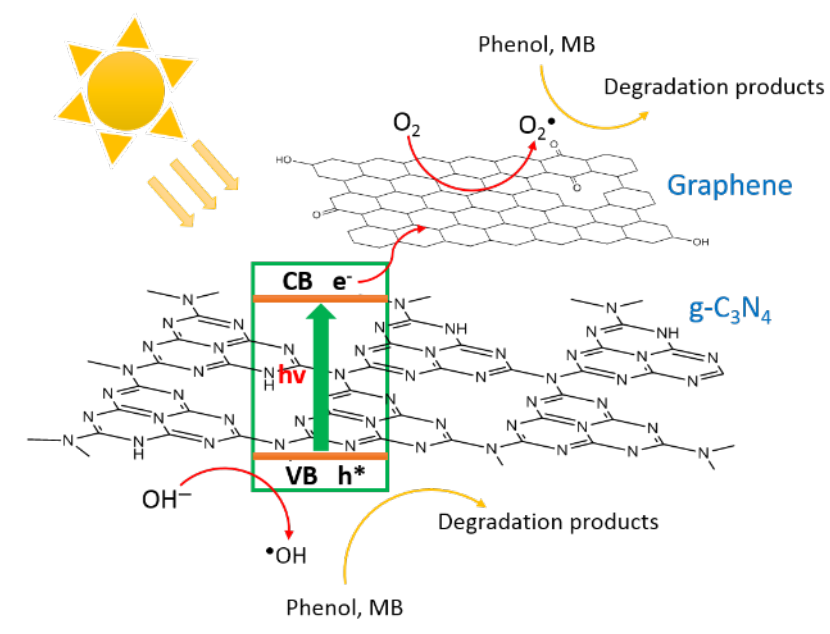

Fig. 11 Photodegradation of organics on g- $\mathrm{C}_{3} \mathrm{~N}_{4}$ /graphene hybrids.

\subsection{Roles of graphene for enhanced photocatalysis}

Pure g- $\mathrm{C}_{3} \mathrm{~N}_{4}$ is more sensitive to UV and severely suffered from the drawbacks of quick recombination of the photogenerated electron/hole pairs [38]. Ge et al. [52] introduced multiwalled carbon nanotubes (MWCNTs) as a channel to simulate the photogenerated electrons transferring from g- $\mathrm{C}_{3} \mathrm{~N}_{4}$ surface to MWCNTs, which presented a lower Fermi level than g$\mathrm{C}_{3} \mathrm{~N}_{4}$. The g- $\mathrm{C}_{3} \mathrm{~N}_{4} / \mathrm{MWCNTs}$ hybrids presented a higher photocurrent intensity and a better photocatalytic activity toward hydrogen evolution. Sun and co-workers [40] synthesized g$\mathrm{C}_{3} \mathrm{~N}_{4} /$ carbon sphere (CS) composites via a hydrothermal method. The hydrothermal process was able to modify the crystal structure of $\mathrm{g}-\mathrm{C}_{3} \mathrm{~N}_{4}$ and improve phenol adsorption. The interfaces between g- $\mathrm{C}_{3} \mathrm{~N}_{4}$ and CS effectively prevented the recombination of electron/hole pairs and enhance light absorption under visible light region. Graphene possesses a similar layer structure with g- $\mathrm{C}_{3} \mathrm{~N}_{4}$ and optical chemical and electronic properties [15, 31, 55]. The mechanism of graphene promoted photodegradation of MB and phenol was illustrated in Fig. 11. Introduced graphene could significantly improve the optical absorption under visible light and increased the photo-oxidative degradation as shown in Figs. 9 and 10. The CN/graphene hybrids facilitated the excited electrons from the valence band of $g-\mathrm{C}_{3} \mathrm{~N}_{4}$ to the conduction band followed by a fast transportation to the graphene sheet, which results in a lower recombination rate between the photo-motivated holes and electrons [38, 39]. The holes would abstract one electron from the target organic compound (MB or phenol) and the chemically unstable organic substance was quickly transferred to degradation products. Besides, the extra electron, which was transported into the $\mathrm{sp}^{2}$ hybridized carbon honeycomb, might be restricted at the Lewis basic sites (quinone and ketonic groups, $-\mathrm{C}=\mathrm{O}$ ) of the 
reduced graphene oxide but can transfer to the water and oxygen molecules from graphene basal plane and the defective sites (vacancy and edges) to form active radicals for attacking MB and phenol to degradation products [15, 40]. Additionally, it was well reported that carbonaceous intermediates could improve phenol and visible light absorption through dyesensitization, then enhancing the photooxidation process $[40,56]$.

\section{Conclusions}

CN-graphene composites were prepared by a simple thermal condensation of melamine with $\mathrm{GO}$ at $550{ }^{\circ} \mathrm{C}$. Compared to pristine carbon nitride, enhanced photocatalytic oxidation of MB and phenol under visible and UV-visible light was achieved. The introduced graphene was able to modulate both the adsorption capacity and optical absorption in visible light radiation. Increased level of rGO can significantly improve the adsorption of MB on CN-G samples, extend the visible light absorption, and then enhance the visible light photodegradation of MB. The hybrid photocatalysts were also able to efficiently degrade phenol solutions. Both catalyst loading and initial phenol concentration showed significant effects on phenol removal under UV-visible light. This study provided an efficient and green photocatalyst for degradation of dye and other organic pollutants, and can contribute to the development of sustainable remediation technologies.

\section{Acknowledgements}

This work was financially supported by Australian Research Council (DP130101319). The authors acknowledge the use of equipment, scientific and technical assistance of the Curtin University Electron Microscope Facility which has been partially funded by the University, State and Commonwealth Governments. H. S. thanks the support from Curtin Research Fellowship.

\section{References}

[1] E. Neyens, J. Baeyens, A review of classic Fenton's peroxidation as an advanced oxidation technique, J. Hazard. Mater., 98 (2003) 33-50.

[2] E. Chamarro, A. Marco, S. Esplugas, Use of Fenton reagent to improve organic chemical biodegradability, Water Res., 35 (2001) 1047-1051.

[3] S.K. Ling, S.B. Wang, Y.L. Peng, Oxidative degradation of dyes in water using $\mathrm{Co}^{2+} / \mathrm{H}_{2} \mathrm{O}_{2}$ and $\mathrm{Co}^{2+} /$ peroxymonosulfate, J. Hazard. Mater., 178 (2010) 385-389. 
[4] J. Jeong, J. Yoon, pH effect on $\mathrm{OH}$ radical production in photo/ferrioxalate system, Water Res., 39 (2005) 2893-2900.

[5] H.Q. Sun, S.Z. Liu, G.L. Zhou, H.M. Ang, M.O. Tade, S.B. Wang, Reduced graphene oxide for catalytic oxidation of aqueous organic pollutants, ACS Appl. Mater. Interfaces, 4 (2012) 5466-5471.

[6] H.Q. Sun, Y.X. Wang, S.Z. Liu, L. Ge, L. Wang, Z.H. Zhu, S.B. Wang, Facile synthesis of nitrogen doped reduced graphene oxide as a superior metal-free catalyst for oxidation, Chem. Commun., 49 (2013) 9914-9916.

[7] H. Sun, S. Liu, S. Liu, S. Wang, A comparative study of reduced graphene oxide modified $\mathrm{TiO}_{2}, \mathrm{ZnO}$ and $\mathrm{Ta}_{2} \mathrm{O}_{5}$ in visible light photocatalytic/photochemical oxidation of methylene blue, Appl. Catal. B, 146 (2014) 162-168.

[8] S.C. Yan, Z.S. Li, Z.G. Zou, Photodegradation performance of g- $\mathrm{C}_{3} \mathrm{~N}_{4}$ fabricated by directly heating melamine, Langmuir, 25 (2009) 10397-10401.

[9] H.Q. Sun, Y. Bai, H.J. Liu, W.Q. Jin, N.P. Xu, G.J. Chen, B.Q. Xu, Mechanism of nitrogen-concentration dependence on $\mathrm{pH}$ value: Experimental and theoretical studies on nitrogen-doped $\mathrm{TiO}_{2}$, J. Phys. Chem. C, 112 (2008) 13304-13309.

[10] E. Leyva, E. Moctezuma, M.G. Ruiz, L. Torres-Martinez, Photodegradation of phenol and 4-chlorophenol by $\mathrm{BaO}-\mathrm{Li}_{2} \mathrm{O}-\mathrm{TiO}_{2}$ catalysts, Catal. Today, 40 (1998) 367-376.

[11] I.W. Mwangi, J.C. Ngila, P. Ndungu, T.A.M. Msagati, J.N. Kamau, Immobilized Fe (III)-doped titanium dioxide for photodegradation of dissolved organic compounds in water, Environ. Sci. Pollut. Res., 20 (2013) 6028-6038.

[12] Z.J. Wu, W. Huang, K.K. Cui, Z.F. Gao, P. Wang, Sustainable synthesis of metalsdoped $\mathrm{ZnO}$ nanoparticles from zinc-bearing dust for photodegradation of phenol, J. Hazard. Mater., 278 (2014) 91-99.

[13] X.C. Wang, K. Maeda, A. Thomas, K. Takanabe, G. Xin, J.M. Carlsson, K. Domen, M. Antonietti, A metal-free polymeric photocatalyst for hydrogen production from water under visible light, Nat. Mater., 8 (2009) 76-80.

[14] Y. Wang, X.C. Wang, M. Antonietti, Polymeric graphitic carbon nitride as a heterogeneous organocatalyst: From photochemistry to multipurpose catalysis to sustainable chemistry, Angew. Chem. Int. Ed., 51 (2012) 68-89.

[15] X.H. Li, J.S. Chen, X.C. Wang, J.H. Sun, M. Antonietti, Metal-free activation of dioxygen by graphene/g- $\mathrm{C}_{3} \mathrm{~N}_{4}$ nanocomposites: Functional dyads for selective oxidation of saturated hydrocarbons, J. Am. Chem. Soc., 133 (2011) 8074-8077. 
[16] B.H. Long, Z.X. Ding, X.C. Wang, Carbon nitride for the selective oxidation of aromatic alcohols in water under visible light, ChemSusChem, 6 (2013) 2074-2078.

[17] Y. Zheng, J. Liu, J. Liang, M. Jaroniec, S.Z. Qiao, Graphitic carbon nitride materials: controllable synthesis and applications in fuel cells and photocatalysis, Energ. Environ. Sci., 5 (2012) 6717-6731.

[18] H.J. Yan, Soft-templating synthesis of mesoporous graphitic carbon nitride with enhanced photocatalytic $\mathrm{H}_{2}$ evolution under visible light, Chem. Commun., 48 (2012) 34303432.

[19] P. Niu, L.L. Zhang, G. Liu, H.M. Cheng, Graphene-like carbon nitride nanosheets for improved photocatalytic activities, Adv. Funct. Mater., 22 (2012) 4763-4770.

[20] X.F. Chen, J.S. Zhang, X.Z. Fu, M. Antonietti, X.C. Wang, Fe-g- $\mathrm{C}_{3} \mathrm{~N}_{4}$-catalyzed oxidation of benzene to phenol using hydrogen peroxide and visible light, J. Am. Chem. Soc., 131 (2009) 11658-11659.

[21] X.C. Wang, X.F. Chen, A. Thomas, X.Z. Fu, M. Antonietti, Metal-containing carbon nitride compounds: A new functional organic-metal hybrid material, Adv. Mater., 21 (2009) 1609-1610.

[22] Z.X. Ding, X.F. Chen, M. Antonietti, X.C. Wang, Synthesis of transition metal-modified carbon nitride polymers for selective hydrocarbon oxidation, ChemSusChem, 4 (2011) 274281.

[23] S.C. Yan, S.B. Lv, Z.S. Li, Z.G. Zou, Organic-inorganic composite photocatalyst of g$\mathrm{C}_{3} \mathrm{~N}_{4}$ and TaON with improved visible light photocatalytic activities, Dalton Transact., 39 (2010) 1488-1491.

[24] C.C. Han, L. Ge, C.F. Chen, Y.J. Li, X.L. Xiao, Y.N. Zhang, L.L. Guo, Novel visible light induced $\mathrm{Co}_{3} \mathrm{O}_{4}-\mathrm{g}-\mathrm{C}_{3} \mathrm{~N}_{4}$ heterojunction photocatalysts for efficient degradation of methyl orange, Appl. Catal. B, 147 (2014) 546-553.

[25] Y.L. Meng, J. Shen, D. Chen, G. Xin, Photodegradation performance of methylene blue aqueous solution on $\mathrm{Ag} / \mathrm{g}-\mathrm{C}_{3} \mathrm{~N}_{4}$ catalyst, Rare Metals, 30 (2011) 276-279.

[26] L. Ge, C.C. Han, J. Liu, Y.F. Li, Enhanced visible light photocatalytic activity of novel polymeric g-C3N4 loaded with Ag nanoparticles, Appl. Catal. A, 409 (2011) 215-222.

[27] S.C. Yan, Z.S. Li, Z.G. Zou, Photodegradation of rhodamine B and methyl orange over boron-doped g- $\mathrm{C}_{3} \mathrm{~N}_{4}$ under visible light irradiation, Langmuir, 26 (2010) 3894-3901.

[28] Y. Wang, J.S. Zhang, X.C. Wang, M. Antonietti, H.R. Li, Boron- and fluorinecontaining mesoporous carbon nitride polymers: Metal-free catalysts for cyclohexane oxidation, Angew. Chem. Int. Ed., 49 (2010) 3356-3359. 
[29] S.J. Guo, S.J. Dong, Graphene nanosheet: synthesis, molecular engineering, thin film, hybrids, and energy and analytical applications, Chem. Soc. Rev., 40 (2011) 2644-2672.

[30] D.S. Su, S. Perathoner, G. Centi, Nanocarbons for the Development of Advanced Catalysts, Chem. Rev., 113 (2013) 5782-5816.

[31] A.K. Geim, K.S. Novoselov, The rise of graphene, Nat. Mater., 6 (2007) 183-191.

[32] S.Z. Liu, H.Q. Sun, S.M. Liu, S.B. Wang, Graphene facilitated visible light photodegradation of methylene blue over titanium dioxide photocatalysts, Chem. Engin. J., 214 (2013) 298-303.

[33] Q.W. Huang, S.Q. Tian, D.W. Zeng, X.X. Wang, W.L. Song, Y.Y. Li, W. Xiao, C.S. Xie, Enhanced photocatalytic activity of chemically bonded $\mathrm{TiO}_{2}$ /graphene composites based on the effective interfacial charge transfer through the C-Ti bond, ACS Catal., 3 (2013) 14771485.

[34] J.Y. Jing, Y. Zhang, W.Y. Li, W.W. Yu, Visible light driven photodegradation of quinoline over $\mathrm{TiO}_{2}$ /graphene oxide nanocomposites, J. Catal., 316 (2014) 174-181.

[35] H.Q. Sun, S.B. Wang, Research advances in the synthesis of nanocarbon-based photocatalysts and their applications for photocatalytic conversion of carbon dioxide to hydrocarbon fuels, Energy Fuels, 28 (2014) 22-36.

[36] W.C. Peng, X.Y. Li, Synthesis of a sulfur-graphene composite as an enhanced metal-free photocatalyst, Nano Res., 6 (2013) 286-292.

[37] Y.J. Zhang, T. Mori, L. Niu, J.H. Ye, Non-covalent doping of graphitic carbon nitride polymer with graphene: controlled electronic structure and enhanced optoelectronic conversion, Energ. Environ. Sci., 4 (2011) 4517-4521.

[38] A.J. Du, S. Sanvito, Z. Li, D.W. Wang, Y. Jiao, T. Liao, Q. Sun, Y.H. Ng, Z.H. Zhu, R. Amal, S.C. Smith, Hybrid graphene and graphitic carbon nitride nanocomposite: Gap opening, electron-hole puddle, interfacial charge transfer, and rnhanced visible light response, J. Am. Chem. Soc., 134 (2012) 4393-4397.

[39] Q.J. Xiang, J.G. Yu, M. Jaroniec, Preparation and enhanced visible-light photocatalytic $\mathrm{H}_{2}$ production activity of graphene/ $\mathrm{C}_{3} \mathrm{~N}_{4}$ composites, J. Phys. Chem. C, 115 (2011) 73557363.

[40] H. Sun, G. Zhou, Y. Wang, A. Suvorova, S. Wang, A new metal-free carbon hybrid for enhanced photocatalysis, ACS Appl. Mater. Interfaces, 6 (2014) 16745-16754.

[41] W.S. Hummers, R.E. Offeman, Preparation of graphitic oxide, J. Am. Chem. Soc., 80 (1958) 1339-1339. 
[42] J.H. Liu, T.K. Zhang, Z.C. Wang, G. Dawson, W. Chen, Simple pyrolysis of urea into graphitic carbon nitride with recyclable adsorption and photocatalytic activity, J. Mater. Chem., 21 (2011) 14398-14401.

[43] A. Thomas, A. Fischer, F. Goettmann, M. Antonietti, J.O. Muller, R. Schlogl, J.M. Carlsson, Graphitic carbon nitride materials: variation of structure and morphology and their use as metal-free catalysts, J. Mater. Chem., 18 (2008) 4893-4908.

[44] X.C. Wang, S. Blechert, M. Antonietti, Polymeric graphitic carbon nitride for heterogeneous photocatalysis, ACS Catal., 2 (2012) 1596-1606.

[45] Y.J. Cui, J.H. Huang, X.Z. Fu, X.C. Wang, Metal-free photocatalytic degradation of 4chlorophenol in water by mesoporous carbon nitride semiconductors, Catal. Sci. Technol., 2 (2012) 1396-1402.

[46] G.G. Zhang, J.S. Zhang, M.W. Zhang, X.C. Wang, Polycondensation of thiourea into carbon nitride semiconductors as visible light photocatalysts, J. Mater. Chem., 22 (2012) 8083-8091.

[47] G.Z. Liao, S. Chen, X. Quan, H.T. Yu, H.M. Zhao, Graphene oxide modified g- $\mathrm{C}_{3} \mathrm{~N}_{4}$ hybrid with enhanced photocatalytic capability under visible light irradiation, J. Mater. Chem., 22 (2012) 2721-2726.

[48] F. Dong, L. Wu, Y. Sun, M. Fu, Z. Wu, S. Lee, Efficient synthesis of polymeric g- $\mathrm{C}_{3} \mathrm{~N}_{4}$ layered materials as novel efficient visible light driven photocatalysts, J. Mater. Chem., 21 (2011) 15171-15174.

[49] M. Groenewolt, M. Antonietti, Synthesis of $\mathrm{g}-\mathrm{C}_{3} \mathrm{~N}_{4}$ nanoparticles in mesoporous silica host matrices, Adv. Mater., 17 (2005) 1789-1790.

[50] Y.J. Zhang, A. Thomas, M. Antonietti, X.C. Wang, Activation of carbon nitride solids by protonation: Morphology changes, enhanced ionic conductivity, and photoconduction experiments, J. Am. Chem. Soc., 131 (2009) 50-51.

[51] X.L. Li, H.L. Wang, J.T. Robinson, H. Sanchez, G. Diankov, H.J. Dai, Simultaneous nitrogen doping and reduction of graphene oxide, J. Am. Chem. Soc., 131 (2009) 1593915944.

[52] L. Ge, C.C. Han, Synthesis of MWNTs/g-C ${ }_{3} \mathrm{~N}_{4}$ composite photocatalysts with efficient visible light photocatalytic hydrogen evolution activity, Appl. Catal. B, 117 (2012) 268-274. [53] Y.Q. Sun, C. Li, Y.X. Xu, H. Bai, Z.Y. Yao, G.Q. Shi, Chemically converted graphene as substrate for immobilizing and enhancing the activity of a polymeric catalyst, Chem. Commun., 46 (2010) 4740-4742. 
[54] F. He, G. Chen, Y.G. Yu, S. Hao, Y.S. Zhou, Y. Zheng, Facile approach to synthesize gPAN/g- $\mathrm{C}_{3} \mathrm{~N}_{4}$ composites with enhanced photocatalytic $\mathrm{H}_{2}$ evolution activity, ACS Appl. Mater. Interfaces, 6 (2014) 7171-7179.

[55] M.J. Allen, V.C. Tung, R.B. Kaner, Honeycomb carbon: A review of graphene, Chem. Rev., 110 (2010) 132-145.

[56] Y.B. Wang, J.D. Hong, W. Zhang, R. Xu, Carbon nitride nanosheets for photocatalytic hydrogen evolution: remarkably enhanced activity by dye sensitization, Catal. Sci. Technol., 3 (2013) 1703-1711. 